\title{
An Active Site Inhibitor Induces Conformational Penalties for ACE2 Recognition by the Spike Protein of SARS-CoV-2
}

Billy J Williams-Noonan ${ }^{1}$, Nevena Todorova ${ }^{1}$, Ketav Kulkarni ${ }^{2}$, Marie-Isabel Aguilar ${ }^{2}$ and Irene Yarovsky $^{1 *}$

\author{
${ }^{1}$ RMIT University, School of Engineering, Melbourne, VIC, 3001, Australia \\ ${ }^{2}$ Department of Biochemistry and Molecular Biology, Monash University, Clayton, VIC, 3800, Australia.
}

\section{Supplementary Information}

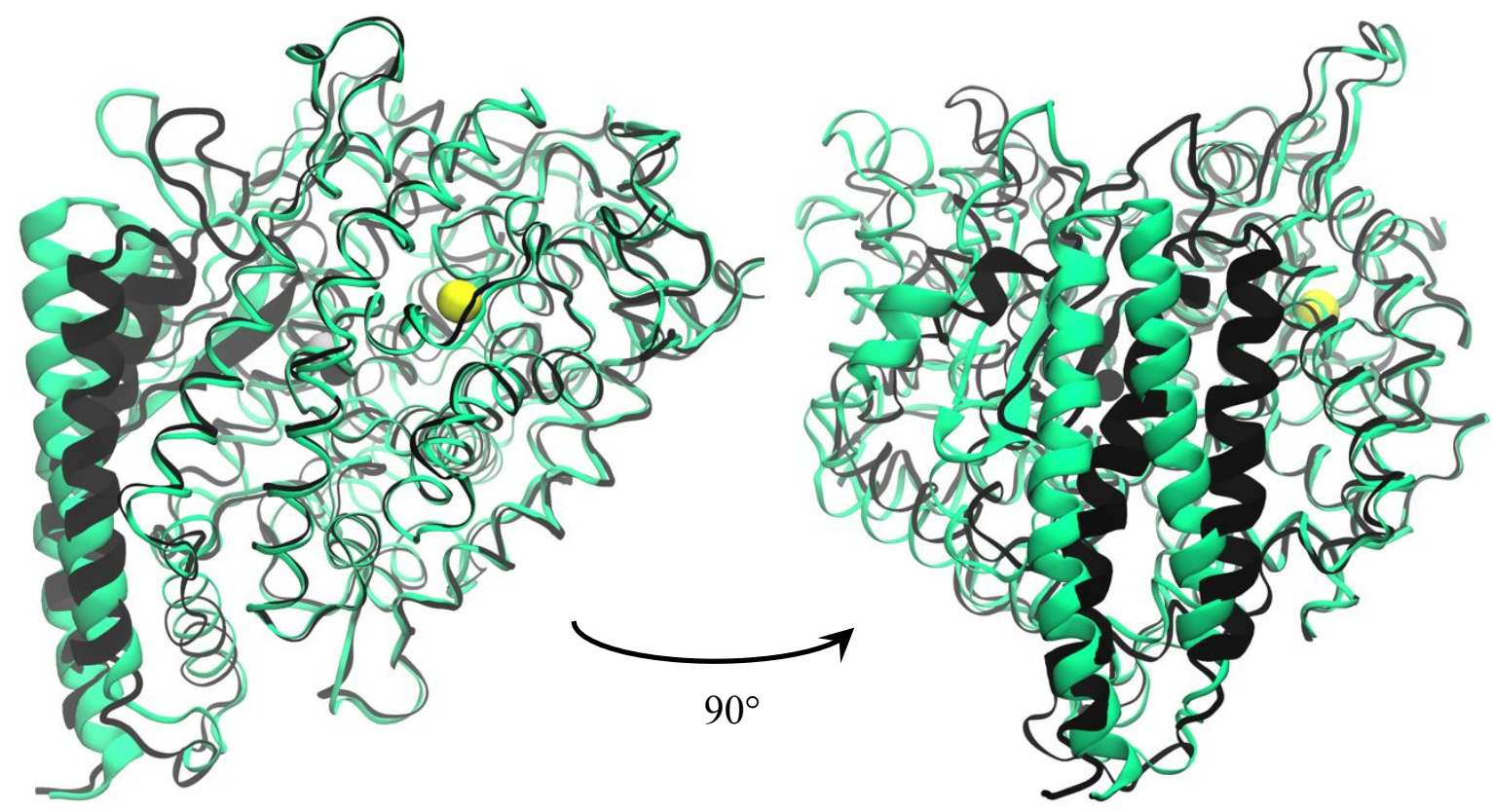

Figure S1. Superimposed structures of ACE2 from PDB-ID: 6M0J (green) and ACE2 from PDB-ID: 1R4L (black). Motifs that align well are given a wire representation while those with noticeable conformational change are shown as a cartoon representation. Catalytic $\mathrm{Zn}$ and $\mathrm{Cl}$ ions are shown as grey and yellow spheres. A 90 degrees view of the superimposed ACE2 proteins is shown on the right. 
Table S1. Ensemble RMSD \pm 1 SD $(\AA)$ of ACE2 and key ACE2 motifs using both 2 fs and 5 fs timesteps. The conformational changes to the s-protein binding site residues are consistent with both timesteps.

\begin{tabular}{|c|c|c|c|c|c|c|}
\hline $\begin{array}{l}\text { Timestep } \\
\text { (fs) }\end{array}$ & $\begin{array}{l}\text { Simulation } \\
\text { System }\end{array}$ & $\begin{array}{l}\text { Total } \\
\text { ACE2 }\end{array}$ & $\begin{array}{c}\text { S-Protein } \\
\text { Binding Site } \\
\text { of ACE2 a }^{\text {A }}\end{array}$ & $\begin{array}{c}\text { Key Interacting } \\
\text { Residues from } \\
\text { ACE2 }^{\text {b }}\end{array}$ & $\begin{array}{c}\text { Non-interacting } \\
\text { Residues of ACE2 } \\
\text { within S-protein } \\
\text { Binding Site } \\
\text { c }\end{array}$ & $\begin{array}{l}\text { ACE2 residues not } \\
\text { belonging to the } S \text { - } \\
\text { protein Binding Site }\end{array}$ \\
\hline $5^{\mathrm{d}}$ & Apo-ACE2 & $2.8 \pm 0.4$ & $2.5 \pm 0.6$ & $2.8 \pm 0.4$ & $2.9 \pm 0.7$ & $2.9 \pm 0.6$ \\
\hline $2^{\mathrm{e}}$ & Apo-ACE2 & $2.6 \pm 0.3$ & $2.5 \pm 0.4$ & $2.2 \pm 0.4$ & $2.5 \pm 0.5$ & $2.6 \pm 0.3$ \\
\hline $5^{\mathrm{d}}$ & MLN/ACE2 & $4.8 \pm 0.4$ & $6.7 \pm 0.8$ & $5.0 \pm 0.6$ & $7.8 \pm 1.0$ & $4.4 \pm 0.3$ \\
\hline $2^{\mathrm{e}}$ & MLN/ACE2 & $5.1 \pm 0.1$ & $6.8 \pm 0.3$ & $5.0 \pm 0.4$ & $7.9 \pm 0.3$ & $4.7 \pm 0.1$ \\
\hline
\end{tabular}

a,b,c As defined in Table 1 of the main text

${ }^{\mathrm{d}}$ Performed as three $500 \mathrm{~ns}$ replicate simulations, and RMSD values are an average of the three replicates

e Performed as one $500 \mathrm{~ns}$ simulation, and the RMSD values are the ensemble average

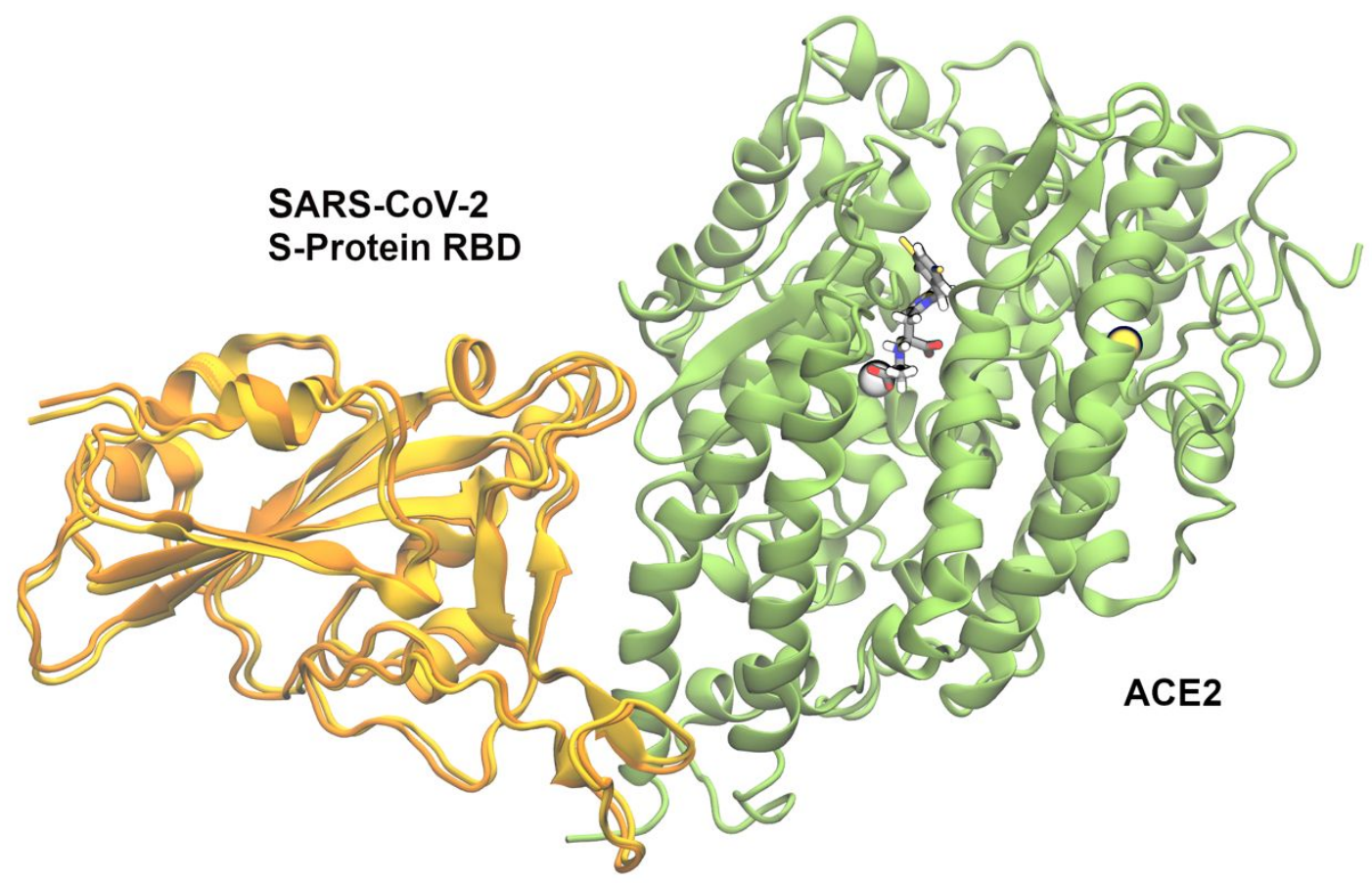

Figure S2. Alternate s-protein orientations for System 4. The MLN/ACE2/s-protein model is given a ribbon representation. The s-protein rotated $+5^{\circ}$ is in orange, and the s-protein rotated $-5^{\circ}$ is in yellow. Catalytic site $\mathrm{Cl}^{-}$is given a yellow sphere representation, and the $\mathrm{Zn}$ is colour silver. Active site inhibitor MLN (MLN-4760) is shown as sticks. 
Table S2. Minimum interaction distances from our initial model of the MLN/ACE2/s-protein complex and over the ensemble of structures of system (4), measured in $\AA$. Also included are the minimum interaction lengths from the production simulations of the MLN/ACE2/s-protein complex where the s-protein is rotated $\pm 5^{\circ}$. Each of the three variants of system (4) are equilibrated for $400 \mathrm{~ns}$ before determining minimum interaction distances from an ensemble. Clearly, all key interactions are present in each model after $400 \mathrm{~ns}$.

\begin{tabular}{|c|c|c|c|c|c|c|c|}
\hline $\begin{array}{c}\text { Interaction } \\
\text { Type }\end{array}$ & $\begin{array}{c}\text { Subunit 1: } \\
\text { ACE2 }\end{array}$ & $\begin{array}{l}\text { Subunit 2: } \\
\text { S-protein } \\
\text { RBD }\end{array}$ & $\begin{array}{l}\text { Crystallographic } \\
\text { Interaction Length } \\
\text { (PDB-ID: 6M0J) }\end{array}$ & $\begin{array}{c}\text { Minimum } \\
\text { Interaction } \\
\text { Length } \\
\text { (Initial }^{\text {Model) }}{ }^{\mathrm{a}} \\
\end{array}$ & $\begin{array}{c}\text { Minimum } \\
\text { Interaction } \\
\text { Length } \\
(\mathrm{s}-\text { protein } \\
\left.\text { rotated }+5^{\circ}\right)^{\mathrm{b}}\end{array}$ & $\begin{array}{c}\text { Minimum } \\
\text { Interaction } \\
\text { Length } \\
(\text { s-protein } \\
\left.\text { rotated - } 5^{\circ}\right)^{b}\end{array}$ & $\begin{array}{c}\text { Mean Signed } \\
\text { Deviation } \\
\text { from Initial } \\
\text { Model c }^{c}\end{array}$ \\
\hline \multirow[t]{13}{*}{ H-Bonds } & Q-24 (OE1) & N-487 (ND2) & 2.6 & 2.7 & 2.6 & 2.7 & -0.1 \\
\hline & D-30 (OD2) & K-417 (NZ) & 3.0 & 2.5 & 2.5 & 2.5 & 0.0 \\
\hline & E-35 (OE2) & Q-493 (NE2) & 2.8 & 2.7 & 1.6 & 1.6 & -1.1 \\
\hline & E-37 (OE2) & Y-505 (OH) & 3.2 & 1.6 & 2.6 & 2.5 & 1.0 \\
\hline & D-38 (OD2) & Y-449 (OH) & 2.7 & 1.5 & 2.6 & 2.4 & 1.0 \\
\hline & $\mathrm{Y}-41(\mathrm{OH})$ & $\mathrm{T}-500(\mathrm{OG} 1)$ & 2.6 & 2.4 & 2.6 & 2.6 & 0.2 \\
\hline & $\mathrm{Y}-41(\mathrm{OH})$ & $\mathrm{N}-501(\mathrm{~N})$ & 3.7 & 2.6 & 3.7 & 3.5 & 0.9 \\
\hline & Q-42 (NE2) & $\mathrm{G}-446(\mathrm{O})$ & 3.3 & 3.3 & 2.7 & 3.1 & -0.4 \\
\hline & Q-42 (NE2) & $\mathrm{Y}-449(\mathrm{OH})$ & 3.0 & 2.5 & 1.9 & 2.0 & -0.6 \\
\hline & $\mathrm{Y}-83(\mathrm{OH})$ & Y-489 (OH) & 3.5 & 1.6 & 2.0 & 2.9 & 0.9 \\
\hline & $\mathrm{Y}-83(\mathrm{OH})$ & N-487 (OD1) & 2.7 & 1.7 & 2.9 & 2.8 & 1.2 \\
\hline & $\mathrm{K}-353(\mathrm{O})$ & G-502 (N) & 2.8 & 1.6 & 2.6 & 2.5 & 0.9 \\
\hline & R-393 (CZ) & $\mathrm{Y}-505(\mathrm{OH})$ & 4.8 & 3.6 & 3.5 & 3.9 & 0.0 \\
\hline \multirow[t]{2}{*}{ Salt Bridges } & $\mathrm{D}-30(\mathrm{OD} 1)$ & K-417 (NZ) & 3.9 & 2.5 & 2.5 & 2.5 & 0.0 \\
\hline & D-30 (OD2) & K-417 (NZ) & 3.0 & 2.5 & 2.5 & 2.5 & 0.0 \\
\hline
\end{tabular}

${ }^{a}$ Minimum interaction distance from triplicate $1.0 \mu \mathrm{s} \mathrm{MD} \mathrm{trajectories,} \mathrm{discarding} \mathrm{the} \mathrm{first} 400 \mathrm{~ns}$ as an additional equilibration phase (first $400 \mathrm{~ns}$ of each replicate not included in distance calculation). Total simulation time for distance calculation is $1.8 \mu \mathrm{s}$.

${ }^{\mathrm{b}}$ Minimum interaction distance from a $500 \mathrm{~ns}$ MD trajectory, discarding the first $400 \mathrm{~ns}$ as an additional equilibration phase (first $400 \mathrm{~ns}$ not included in distance calculation). Total simulation time for distance calculation is $100 \mathrm{~ns}$.

${ }^{c}$ Average (directed) deviation from the initial model (system 4) for s-protein rotated $\pm 5^{\circ}$ in the Z-direction. 


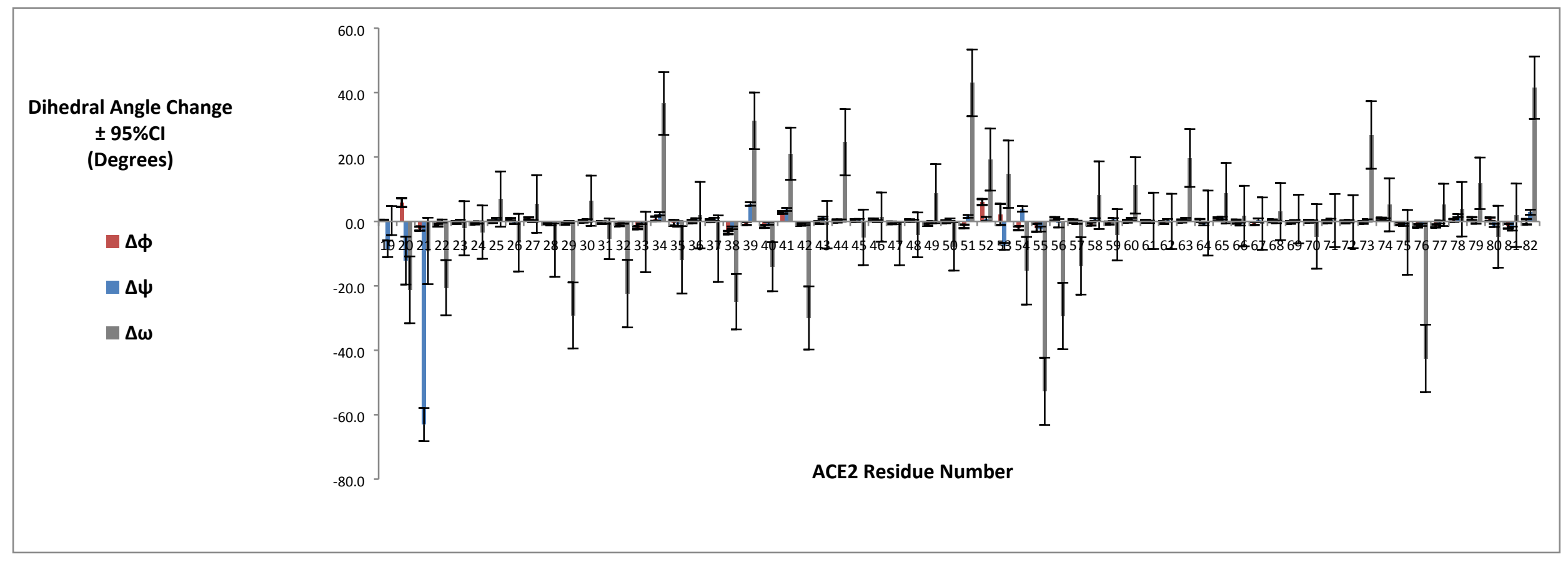

Figure S3. Change in backbone $\varphi, \psi$ and $\omega$ angles of the ACE2 N-terminal, $\alpha-1$ and $\alpha-2$ helices when MLN is bound to ACE2. All values are a mean $\pm 95 \%$ CI from the combined MD ensembles of systems (1) and (2) (see the main text). 
Table S3. The change in the backbone $\varphi, \psi$ and $\omega$ angles (in degrees) of the residues comprising the $\alpha-1$ to $\alpha-2$ helices of ACE2, when MLN is bound to ACE2. All values are measured in degrees and are an ensemble average $\pm 95 \% \mathrm{CI}$.

\begin{tabular}{|c|c|c|c|c|c|c|}
\hline Residue & $\Delta \varphi$ & $95 \% \mathrm{CI}$ & $\Delta \psi$ & $95 \% \mathrm{CI}$ & $\Delta \omega$ & $95 \% \mathrm{CI}$ \\
\hline 19 & 0.4 & 0.1 & -8.5 & 2.6 & 0.3 & 4.5 \\
\hline 20 & 5.9 & 1.4 & -12.2 & 7.4 & -21.2 & 10.4 \\
\hline 21 & -2.2 & 0.6 & -63.1 & 5.1 & -9.2 & 10.3 \\
\hline 22 & -1.2 & 0.4 & 0.1 & 0.5 & -20.6 & 8.6 \\
\hline 23 & -0.4 & 0.4 & 0.2 & 0.4 & -2.1 & 8.4 \\
\hline 24 & -0.5 & 0.4 & -0.3 & 0.4 & -3.3 & 8.3 \\
\hline 25 & -0.1 & 0.4 & 0.8 & 0.3 & 6.9 & 8.6 \\
\hline 26 & 0.6 & 0.4 & -0.5 & 0.4 & -6.6 & 8.9 \\
\hline 27 & 0.9 & 0.4 & 0.1 & 0.4 & 5.4 & 8.9 \\
\hline 28 & -0.5 & 0.4 & -0.9 & 0.3 & -8.9 & 8.3 \\
\hline 29 & -0.6 & 0.4 & -0.1 & 0.3 & -29.2 & 10.2 \\
\hline 30 & 0.1 & 0.4 & 0.3 & 0.4 & 6.4 & 7.8 \\
\hline 31 & -0.4 & 0.4 & -0.4 & 0.4 & -5.4 & 6.3 \\
\hline 32 & -1.1 & 0.4 & -0.4 & 0.4 & -22.4 & 10.5 \\
\hline 33 & -2.0 & 0.4 & -0.5 & 0.4 & -6.4 & 9.4 \\
\hline 34 & 0.8 & 0.7 & 2.4 & 0.5 & 36.6 & 9.7 \\
\hline 35 & -0.4 & 0.9 & -0.8 & 0.9 & -11.9 & 10.5 \\
\hline 36 & -0.1 & 0.5 & 0.5 & 0.5 & 2.0 & 10.3 \\
\hline 37 & 0.2 & 0.4 & 0.6 & 0.5 & -8.5 & 10.3 \\
\hline 38 & -3.5 & 0.5 & -2.0 & 0.4 & -24.9 & 8.6 \\
\hline 39 & -0.6 & 0.5 & 5.4 & 0.5 & 31.2 & 8.8 \\
\hline 40 & -1.6 & 0.4 & -0.6 & 0.4 & -14.1 & 7.6 \\
\hline 41 & 2.8 & 0.4 & 3.7 & 0.5 & 21.0 & 8.1 \\
\hline 42 & -0.9 & 0.4 & -0.8 & 0.4 & -30.0 & 9.8 \\
\hline 43 & -0.4 & 0.4 & 1.1 & 0.4 & -1.0 & 7.4 \\
\hline 44 & 0.1 & 0.4 & 0.3 & 0.3 & 24.6 & 10.3 \\
\hline 45 & 0.3 & 0.4 & 0.4 & 0.4 & -4.9 & 8.6 \\
\hline 46 & 0.5 & 0.4 & 0.1 & 0.4 & 1.3 & 7.6 \\
\hline 47 & -0.4 & 0.4 & -0.5 & 0.4 & -6.9 & 6.7 \\
\hline 48 & 0.3 & 0.4 & 0.2 & 0.3 & -4.2 & 7.0 \\
\hline 49 & -1.0 & 0.4 & -0.2 & 0.3 & 8.6 & 9.2 \\
\hline 50 & -0.1 & 0.4 & 0.6 & 0.4 & -7.8 & 7.5 \\
\hline 51 & -1.6 & 0.5 & 1.6 & 0.4 & 43.0 & 10.4 \\
\hline 52 & 6.0 & 0.9 & 0.7 & 0.7 & 19.2 & 9.6 \\
\hline 53 & 2.2 & 3.3 & -7.7 & 1.1 & 14.7 & 10.4 \\
\hline 54 & -2.1 & 0.6 & 3.9 & 0.9 & -15.3 & 10.5 \\
\hline 55 & -1.9 & 1.2 & -2.4 & 0.8 & -52.7 & 10.4 \\
\hline 56 & 0.8 & 0.5 & -0.6 & 1.3 & -29.4 & 10.3 \\
\hline 57 & 0.2 & 0.5 & -0.3 & 0.5 & -13.8 & 8.9 \\
\hline 58 & -0.8 & 0.5 & 0.6 & 0.4 & 8.1 & 10.5 \\
\hline 59 & -0.5 & 0.4 & 0.6 & 0.5 & -4.2 & 8.0 \\
\hline 60 & 0.1 & 0.4 & 0.7 & 0.4 & 11.2 & 8.8 \\
\hline 61 & 0.1 & 0.4 & -0.1 & 0.4 & 0.2 & 8.8 \\
\hline
\end{tabular}




$\begin{array}{lcccccc}62 & -0.4 & 0.4 & 0.4 & 0.4 & 0.0 & 8.6 \\ 63 & 0.1 & 0.4 & 0.7 & 0.4 & 19.7 & 9.0 \\ 64 & 0.3 & 0.5 & -0.8 & 0.4 & -0.5 & 10.1 \\ 65 & 0.7 & 0.4 & 1.0 & 0.4 & 8.8 & 9.4 \\ 66 & -0.1 & 0.6 & -0.8 & 0.5 & 1.7 & 9.4 \\ 67 & -0.7 & 0.4 & 0.4 & 0.6 & -0.7 & 8.1 \\ 68 & 0.2 & 0.4 & -0.2 & 0.4 & 3.1 & 8.9 \\ 69 & -0.3 & 0.4 & 0.1 & 0.4 & 0.8 & 7.5 \\ 70 & 0.1 & 0.4 & 0.0 & 0.3 & -4.7 & 10.0 \\ 71 & -0.1 & 0.4 & 0.4 & 0.4 & 0.3 & 8.3 \\ 72 & -0.2 & 0.4 & 0.1 & 0.4 & -0.1 & 8.2 \\ 73 & -0.3 & 0.4 & 0.3 & 0.4 & 26.8 & 10.5 \\ 74 & 0.7 & 0.4 & 0.6 & 0.4 & 5.2 & 8.2 \\ 75 & -0.7 & 0.4 & -1.0 & 0.4 & -6.5 & 10.1 \\ 76 & -1.4 & 0.5 & -1.1 & 0.4 & -42.5 & 10.5 \\ 77 & -1.4 & 0.4 & -0.2 & 0.5 & 5.1 & 6.6 \\ 78 & 0.3 & 0.4 & 1.8 & 0.5 & 3.8 & 8.4 \\ 79 & 0.9 & 0.5 & -0.4 & 0.4 & 11.8 & 8.0 \\ 80 & 0.7 & 0.5 & -1.2 & 0.5 & -4.8 & 9.6 \\ 81 & -1.5 & 0.7 & -2.2 & 0.6 & 1.9 & 9.8 \\ 82 & -0.1 & 0.8 & 2.9 & 0.8 & 41.5 & 9.7\end{array}$

Table S4. MM-PBSA analysis to determine s-protein binding enthalpy values for the ACE2/s-protein complex and the MLN/ACE2/s-protein complex. All energy values are reported in $\mathrm{kJ} \mathrm{mol}^{-1}$ and are a mean $\pm \mathrm{SD}$ of the ensemble of values from a respective trajectory. Averages $\pm 1 \mathrm{SD}$ based on the three replicates are located in Table 3 of the main text.

ACE2/s-protein

MLN/ACE2/s-protein

\begin{tabular}{ccccccc}
\hline & Replicate 1 & Replicate 2 & Replicate 3 & Replicate 1 & Replicate 2 & Replicate 3 \\
\hline$\Delta H_{v d W}$ & $-340 \pm 3$ & $-324 \pm 8$ & $-333 \pm 4$ & $-274 \pm 14$ & $-325 \pm 10$ & $-255 \pm 13$ \\
$\Delta H_{\text {elec }}$ & $-68 \pm 1$ & $-70 \pm 1$ & $-67 \pm 1$ & $-62 \pm 2$ & $-69 \pm 2$ & $-54 \pm 1$ \\
$\Delta H_{\text {solv }}$ & $256 \pm 6$ & $250 \pm 6$ & $243 \pm 5$ & $231 \pm 12$ & $260 \pm 12$ & $174 \pm 10$ \\
$S A S A_{\text {en }}$ & $-43 \pm 0.5$ & $-42 \pm 1.0$ & $-42 \pm 1.0$ & $-35 \pm 1.0$ & $-41 \pm 1.0$ & $-29 \pm 1.5$ \\
\hline$\Delta H_{\text {bind }}$ & $-194 \pm 6$ & $-188 \pm 4$ & $-200 \pm 5$ & $-139 \pm 6$ & $-179 \pm 7$ & $-154 \pm 8$ \\
\hline
\end{tabular}

